\title{
Label-Free and Substrate-Free Potentiometric Aptasensing Using Polycation-Sensitive Membrane Electrodes
}

\author{
Jiawang Ding, ${ }^{\dagger, \dagger}$ Yan Chen, ${ }^{\dagger}$ Xuewei Wang, ${ }^{\dagger}$ and Wei Qin* ${ }^{\dagger}{ }^{\dagger}$ \\ P. R. China \\ ॠraduate University of Chinese Academy of Sciences, Beijing 100049, P. R. China
}

${ }^{\dagger}$ Key Laboratory of Coastal Zone Environmental Processes, Yantai Institute of Coastal Zone Research (YIC), Chinese Academy of Sciences (CAS), Shandong Provincial Key Laboratory of Coastal Zone Environmental Processes, YICCAS, Yantai, Shandong 264003,

\section{Supporting Information}

ABSTRACT: A potentiometric label-free and substrate-free (LFSF) aptasensing strategy which eliminates the labeling, separation, and immobilization steps is described in this paper. An aptamer binds specifically to a target molecule via reaction incubation, which could induce a change in the aptamer conformation from a random coil-like configuration to a rigid folded structure. Such a target binding-induced aptamer conformational change effectively prevents the aptamer from electrostatically interacting with the protamine binding domain. This could either shift the response curve for the potentiometric titration of the aptamer with protamine as monitored by a conventional polycation-sensitive membrane electrode or change the current-dependent potential detected by a protamine-conditioned

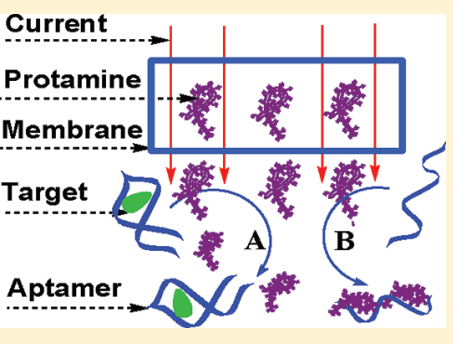
polycation-sensitive electrode with the pulsed current-driven ion fluxes of protamine across the polymeric membrane. Using adenosine triphosphate (ATP) as a model analyte, the proposed concept offers potentiometric detection of ATP down to the submicromolar concentration range and has been applied to the determination of ATP in HeLa cells. In contrast to the current LFSF aptasensors based on optical detection, the proposed strategy allows the LFSF biosensing of aptamer/target binding events in a homogeneous solution via electrochemical transduction. It is anticipated that the proposed strategy will lay a foundation for development of potentiometric sensors for LFSF aptasensing of a variety of analytes where target binding-induced conformational changes such as the formation of folded structures and the opening of DNA hairpin loops are involved.

\begin{abstract}
A ptasensors utilizing aptamers as biorecognition elements have appeared as promising devices for selective and high efficiency detection of a variety of analytes ranging from small ions to large proteins. ${ }^{1,2}$ Compared to the conventional bioreceptors such as antibodies and enzymes, aptamers are much smaller in size, more chemically stable, and less costly. ${ }^{3}$ Various aptasensors based on electrochemical, optical, and mass-sensitive transduction modes have been extensively investigated. ${ }^{4-10}$ In these methods, processes for probe labeling on aptamers, target separation from sample matrix, and aptamer immobilization onto solid substrates are always required. These processes not only involve extra time and cost but also affect the binding affinities between targets and aptamers. In recent years, substantial efforts have been directed to the development of label-free and substrate-free (LFSF) aptasensors, for which the whole measurements can be performed in a homogeneous solution and the labeling, separation, and immobilization steps are effectively eliminated. ${ }^{11,12}$ Currently, most of these LFSF aptasensors are based on fluorescence, UV-visible absorbance, and chemiluminescence detection systems using fluorescent dyes, ${ }^{13}$ gold nanoparticles, ${ }^{14}$ and hemin DNAzymes ${ }^{15}$ as signal reporters. Although electrochemical sensors have potential advantages over other analytical systems in that they are portable, low cost, robust, and easily miniaturized and can operate in turbid media, no electrochemical LFSF aptasensors
\end{abstract}

performed in a homogeneous solution have been reported so far. $^{16-18}$

Over the past few years, significant progress has been made in the development of polymeric membrane polyion-sensitive electrodes for detection of highly charged macromolecules in biological samples. ${ }^{19-22}$ The potentiometric response of such polyion sensors is governed by a nonequilibrium steady-state extraction of the polyion into the organic membrane phase of the electrodes via formation of cooperative ion pairs with lipophilic ion exchangers in the membrane phase. ${ }^{23}$ This response is dependent on the charge density, lipophilicity, and molecular weight of the polyion species. ${ }^{24}$ Aptamers are oligonucleic acid sequences which bind to target molecules with high affinity and specificity. Although nucleic acids including DNA and RNA can be directly measured by using polyanion-sensitive electrodes, the voltage changes have been found rather small due to the poor extraction of these hydrophilic phosphate-rich polyanions into the sensing membranes. ${ }^{25}$ However, aptamers can be indirectly measured by using polycation-sensitive membrane electrodes via electrostatically binding to protamine. Protamine is a group of arginine-rich polycationic proteins extracted from the Salmo-

Received: September 21, 2011

Accepted: January 21, 2012

Published: January 22, 2012 
nidae fish family. ${ }^{26}$ The binding domain of protamine with positively charged guanidinium groups interacts electrostatically with the phosphate groups of DNA, which may form a biochemically inert precipitate. $^{27}$

This paper reports a novel strategy for developing LFSF potentiometric aptasensors, for which protamine is used as an indicator to report the conformational change of aptamer upon recognition of a given target. It will be shown that the target binding-induced aptamer conformational change can effectively prevent the aptamer from electrostatically interacting with the protamine domain, thus dramatically shifting the response curve for the potentiometric titration of the aptamer with protamine as monitored by a conventional polycation-sensitive membrane electrode or changing the current-dependent potential detected by a protamine-conditioned polycationsensitive electrode with the pulsed current-driven ion fluxes of protamine across the polymeric membrane.

\section{EXPERIMENTAL SECTION}

Reagents and Materials. Adenosine-5'-triphosphate (ATP), adenosine-5'-diphosphate (ADP), guanosine-5'-triphosphate (GTP), uridine-5'-triphosphate (UTP), cytidine-5'triphosphate (CTP) disodium salts, adenosine, and adenosine-5'-monophosphate (AMP) were purchased from Shanghai Sangon Biotechnology Co. Ltd. (Shanghai, China). 2-Nitrophenyl octyl ether (o-NPOE), tetradodecylammonium tetrakis(4-chlorophenyl) borate (ETH 500), bis(2-ethylhexyl) sebacate (DOS), tridodecylmethylammonium chloride (TDMAC), and high molecular weight poly(vinyl chloride) (PVC) were purchased from Fluka AG (Buchs, Switzerland). Dinonylnaphthalene sulfonate (DNNS, 50 wt \% solutions in heptane), protamine sulfate salt from herring, heparin sodium salt from bovine intestinal mucosa $(170 \mathrm{U} / \mathrm{mg})$, tetrahydrofuran (THF), and tris(hydroxymethyl)-aminomethane (Tris) were purchased from Sigma. The ATP aptamer was synthesized by Shanghai Sangon Biotechnology Co. Ltd. (Shanghai, China). The aptamer sequence was 5'-ACCTG GGGGA GTATT GCGGA GGAAG GT-3'. All other reagents were analytical grade or better. For all the measurements, $50 \mathrm{mM}$ pH 7.4 Tris$\mathrm{HCl}$ buffer containing $0.12 \mathrm{M} \mathrm{NaCl}$ was used as sample buffer solution. Aqueous solutions were prepared with freshly deionized water $(18.2 \mathrm{M} \Omega \mathrm{cm}$ specific resistance) obtained with a Pall Cascada laboratory water system.

Prior to the measurements, the aptamer was heated at $88{ }^{\circ} \mathrm{C}$ in Tris buffer for $10 \mathrm{~min}$ for aptamer denaturization, which was followed by cooling at room temperature. The heating and cooling steps are necessary to maintain the structure flexibility of the aptamer for high-affinity binding to its target. ${ }^{28,29}$

Membrane Preparation. The membranes of the traditional polycation-sensitive electrodes contained $2.0 \mathrm{wt} \%$ DNNS, 2.0 wt \% ETH 500, 48.0 wt \% o-NPOE, and $48.0 \mathrm{wt}$ $\%$ PVC, while those of the polyanion-sensitive electrodes contained 1.5 wt \% TDMAC, 32.5 wt \% DOS, and 66.0 wt \% PVC. Membranes of $\sim 200 \mu \mathrm{m}$ thickness were obtained by casting a solution of $360 \mathrm{mg}$ of the membrane components dissolved in $3.0 \mathrm{~mL}$ of THF into a glass ring of $3.6 \mathrm{~cm}$ diameter fixed on a glass plate and letting the solvent evaporate overnight. Membrane thicknesses were visually measured with a CX31-32C02 Olympus microscope (Tokyo, Japan). For each ISE, a disk of $7 \mathrm{~mm}$ diameter was punched from the membrane and glued to a plasticized PVC tube ( $6 \mathrm{~mm}$ i.d., $9 \mathrm{~mm}$ o.d.) with THF/PVC slurry. All the electrodes were conditioned overnight in $50 \mathrm{mM} \mathrm{pH} 7.4$ Tris- $\mathrm{HCl}$ buffer solution containing $0.12 \mathrm{M} \mathrm{NaCl}$, which is identical to the inner filling solution.

The membranes for the polycation-selective electrodes with current-controlled release of protamine contained $3.0 \mathrm{wt} \%$ DNNS, 10.0 wt \% ETH 500, 29.0 wt \% PVC, and 58.0 wt \% oNPOE. Protamine solution $(0.05 \mathrm{mg} / \mathrm{mL})$ containing $0.12 \mathrm{M}$ $\mathrm{NaCl}$ in $50 \mathrm{mM}$ Tris- $\mathrm{HCl}$ buffer of $\mathrm{pH} 7.4$ was used as the inner filling solution. The electrodes were conditioned for 3 days in the solution identical to the inner filling solution.

Determination of ATP via Potentiometric Titration. All the measurements of potentiometric titrations were carried out at $20 \pm 2{ }^{\circ} \mathrm{C}$ using a $\mathrm{CHI} 760 \mathrm{C}$ electrochemical workstation (Shanghai Chenhua Apparatus Corporation, China) with an ion-selective electrode (ISE) and an $\mathrm{Ag} / \mathrm{AgCl}$ reference electrode. Measurements of electromotive force (EMF) were performed with stirring in the galvanic cell: $\mathrm{Ag} / \mathrm{AgCl} / 3 \mathrm{M}$ $\mathrm{KCl} /$ /sample solution/ISE membrane/inner filling solution/3 $\mathrm{M} \mathrm{KCl} / \mathrm{AgCl} / \mathrm{Ag}$.

ATP aptamer $(0.66 \mu \mathrm{M})$ was mixed with ATP at various concentrations in $5.0 \mathrm{~mL}$ of Tris buffer. After incubation at room temperature for $30 \mathrm{~min}$, potentiometric titrations of the reaction mixtures using the polycation-sensitive membrane electrode as an end point detector were carried out by successive addition of $5.0 \mu \mathrm{L}$ of $1.0 \mathrm{mg} / \mathrm{mL}$ protamine aqueous solution at a 2 min interval with a syringe pump (TJ-1A syringe pump controller, Hebei, China). Titration curves were obtained by plotting the change in the EMF response vs the concentration of protamine infused. The end points of the potentiomeric titrations used for analyte quantification were determined as the protamine concentration to achieve half of the maximum EMF response $\left(\mathrm{EMF}_{1 / 2, \max }\right)$. By using CTP, UTP, GTP, ADP, and AMP as typical ATP analogues, similar procedures were used for investigation on the specificity of the potentiometric titration system to ATP over its analogues.

Determination of ATP via Current-Controlled Ion Fluxes of Protamine. For potentiometric aptasensing using polycation-sensitive membrane electrodes with current-driven ion fluxes, the measurements were carried out in $50 \mathrm{mM} \mathrm{pH} 7.4$ Tris- $\mathrm{HCl}$ buffer solution containing $0.12 \mathrm{M} \mathrm{NaCl}$ at $20 \pm 2{ }^{\circ} \mathrm{C}$ using a conventional three-electrode system with an ISE electrode, a platinum auxiliary electrode, and an $\mathrm{AgCl} / \mathrm{Ag}$ reference electrode. Experiments were performed on the $\mathrm{CHI}$ $760 \mathrm{C}$ electrochemical workstation. All the measurements were controlled by a macro-command, which executes a series of commands in the specified order. The procedures switching between the galvanostatic and potentiostatic steps were designed according to its Macro Command Dialog box to perform consecutive measurements. The open-circuit potential of the electrode was first recorded for $1 \mathrm{~s}$. Then, an anodic pulsed current of $1 \mu \mathrm{A}$ with a duration of $3 \mathrm{~s}$ was applied for protamine release, and the system was interrogated at zerocurrent for $120 \mathrm{~s}$ to avoid any undesired IR drop. ${ }^{30}$ Finally, a controlled voltage at the open-circuit potential in the absence of analyte was used to refresh the membrane with a recovery time of $60 \mathrm{~s}$ for multiple consecutive measurements. For ATP determination, the ISE potential was first measured in Tris buffer containing $1.67 \mu \mathrm{M}$ of the ATP aptamer to obtain a baseline. Then, $1.67 \mu \mathrm{M}$ of the aptamer was mixed with ATP at various concentrations in Tris buffer. After incubation at room temperature for $30 \mathrm{~min}$, the electrode was immersed into the solution to obtain the response. The current-dependent potential difference $(\Delta E)$ at $3 \mathrm{~s}$ between the baseline and the potential measured after incubation was used for quantification 
of ATP. By using CTP as a typical ATP analogue, similar procedures were used for investigation on the specificity of the aptasensor to ATP over its analogues.

Cellular ATP Assay. The HeLa cells were grown to $90 \%$ confluence in RPMI-1640 medium supplemented with fetal bovine serum (10\%). To eliminate the possible interference from trypsin, the cells were collected after washing with Tris buffer solution. The collected cells were suspended in $1.0 \mathrm{~mL}$ of Tris buffer. Cell lysis of approximately $6 \times 10^{6}$ cells was performed by repeated cycles of freezing (at $-77^{\circ} \mathrm{C}$, dry ice) and thawing (at $37^{\circ} \mathrm{C}$ ), and then the lysed cells were ready for ATP assays. ${ }^{31}$ To remove cell debris in the homogenate, the lysate was centrifuged at $10000 \mathrm{rpm}$ for $5 \mathrm{~min}$. A volume of 3.0 $\mathrm{mL}$ of Tris buffer containing $330 \mu \mathrm{L}$ of freshly lysed cells was used for the ATP assay. Lysed-cell controls were obtained by storing cell lysates for more than $24 \mathrm{~h}$. The ATP detection protocol was the same as mentioned above.

\section{RESULTS AND DISCUSSION}

Our preliminary experiments have ruled out the direct sensing of free or target-bound aptamers using polyanion-sensitive electrodes due to the poor extraction of these hydrophilic phosphate-rich polyanions into the sensing membranes (see Figure S1 in the Supporting Information). In contrast, aptamers can be indirectly measured by using polycation-sensitive membrane electrodes via electrostatically binding to protamine.

The proposed potentiometric LFSF aptasensing strategy is illustrated in Scheme 1. The aptamer binds specifically to the

Scheme 1. Schematic Illustration of Label-Free and Substrate-Free Potentiometric Aptasensing ${ }^{a}$

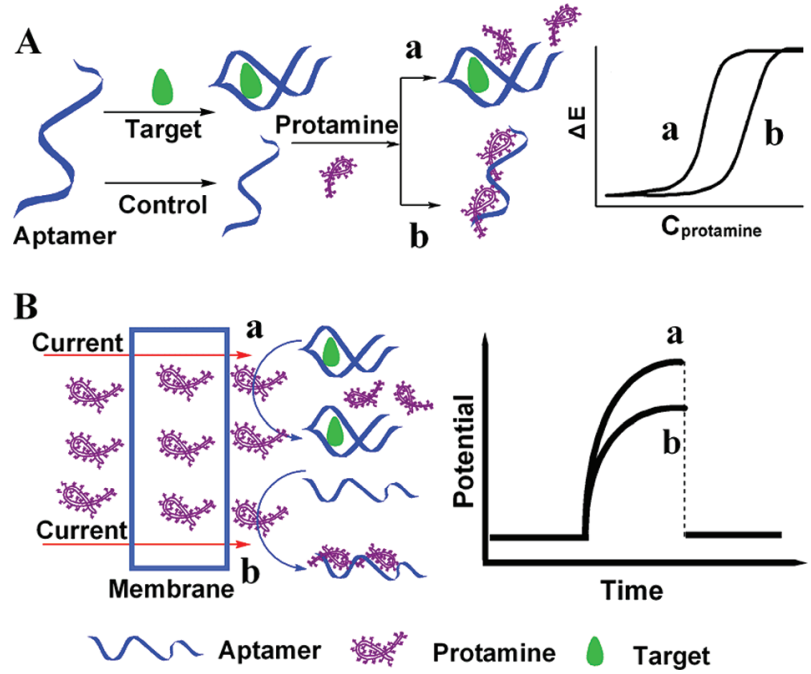

${ }^{a}$ Using (A) a conventional polycation-sensitive membrane electrode based on potentiometric titration with protamine and (B) a protamine-conditioned polycation-sensitive membrane electrode based on current-controlled release of protamine at the samplemembrane interface for (a) a given target and (b) a control assay.

target molecule via reaction incubation, which induces a change in the aptamer conformation from a random coil-like configuration to a folded structure. Such target binding-induced aptamer conformational change decreases the flexibility of the aptamer molecule and effectively prevents the aptamer from electrostatically interacting with the protamine domain, which could either shift the response curve for the potentiometric titration of the aptamer with protamine as monitored by a conventional polycation-sensitive membrane electrode (Scheme 1A) or change the current-dependent potential detected by a protamine-conditioned polycation-sensitive electrode with the pulsed current-driven ion fluxes of protamine across the polymeric membrane (Scheme 1B).

We demonstrate the proof-of-concept aptasensing model using a 27-mer aptamer with a binding constant of $1.67 \times 10^{5}$ $\mathrm{M}^{-1}$ for adenosine-5'-triphosphate (ATP). ${ }^{32-34}$ To understand the electrostatic interaction of the ATP aptamer with protamine, potentiometric titration was carried out using the traditional protamine-sensitive membrane electrode as an endpoint detector. As shown in Figure 1A, the protamine potential
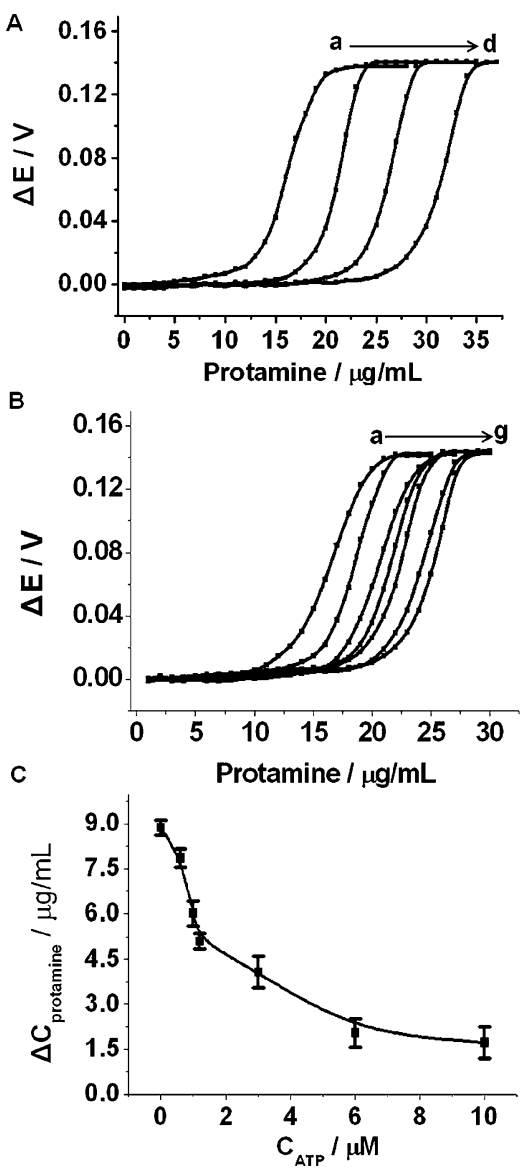

Figure 1. Potentiometric titrations of (A) 0.0 (a), 0.4 (b), 0.8 (c), and $1.2 \mu \mathrm{M}$ aptamer (d) and of (B) $0.0 \mu \mathrm{M}$ aptamer (a) and $0.66 \mu \mathrm{M}$ aptamer in the presence of $6.0(\mathrm{~b}), 3.0(\mathrm{c}), 1.2(\mathrm{~d}), 1.0(\mathrm{e}), 0.6 \mu \mathrm{M}(\mathrm{f})$, and $0.0 \mu \mathrm{M}$ ATP $(\mathrm{g})$ with $1.0 \mathrm{mg} / \mathrm{mL}$ protamine. (C) The calibration curve of the end points obtained from the titration responses for ATP detection. The end point for each titration curve used for analyte quantification was defined as the amount of protamine required to achieve half the total potential response for the analyte titration minus that for the blank titration. Error bars represent 1 standard deviation for three measurements. The potential responses were recorded $120 \mathrm{~s}$ after each addition of $5 \mu \mathrm{L}$ of $1.0 \mathrm{mg} / \mathrm{mL}$ protamine to $5.0 \mathrm{~mL}$ of wellstirred $50 \mathrm{mM}$ pH 7.4 Tris-HCl buffer with a syringe pump.

response curve is shifted to a higher concentration in the presence of the aptamer compared to that of the blank titration; such shifts will be larger with an increase in aptamer concentration. The mass shift can be attributed to electrostatic interaction between the positively charged guanidinium groups of protamine and the negatively charged phosphate groups of 
the aptamer. $^{27}$ The ATP aptamer has a stem-bulge-stem secondary structure, which is typical of aptamers and consists of two small Watson-Crick helices and two G-quartets. Upon annealing, the linear DNA strand may be partially folded into its secondary structure resulting in its ATP recognition conformation. During this process, the partially folded aptamer undergoes a folding equilibrium with a linear aptamer. ${ }^{35}$ In addition, the partially folded aptamer might undergo an unfolding process by binding with protamine. Such flexibility in the aptamer structure facilitates the interaction between the aptamer and protamine. The binding of protamine to the aptamer can be evaluated by recasting the potentiometric titration data in the form of a Scatchard plot (Figure S2A in the Supporting Information). The data analysis treats the aptamer binding domain on a given protamine molecule with an average molecular weight of $4500 \mathrm{Da}$ as an independent binding site. The binding constant was estimated as $2.91( \pm 0.33) \times 10^{6}$ $\mathrm{M}^{-1}$, which is consistent with the results for protamine bindings with DNA. ${ }^{24}$ The number of protamine binding sites per mole of the aptamer was determined to be $3.18( \pm 0.26)$. This binding stoichiometry corresponds to a charge ratio of 2.35:1 (protamine to the ATP aptamer) assuming an average charge of 20 per protamine molecule. ${ }^{36}$ Although the protamineaptamer complex is positively charged, its potential response on the polycation-sensitve electrode is negligible due to the inefficient extraction of the macromolecular complex into the transduction membrane. ${ }^{37}$

Similarly, the potentiometric titration approach was also used to study the effect of the target binding-induced aptamer conformational change on the interaction of aptamer with protamine. The shifts in the titration curves shown in Figure 1B indicate that binding of ATP to the aptamer changes or distorts the aptamer conformation, thus leading to a significant ATPdependent potential response. The potentiometric titration curves are shifted toward lower concentrations with increasing the concentration of ATP, which is due to the inefficient binding of the aptamer-ATP complex to protamine. The binding interaction between protamine and the target-bound aptamer was determined by recasting the titration data of Figure S3 in the Supporting Information in the Scatchard form (Figure S2B in the Supporting Information). The binding constant was estimated as $0.39( \pm 0.26) \times 10^{6} \mathrm{M}^{-1}$, which is much smaller than the protamine-aptamer binding constant. The number of protamine binding sites per mole of the targetbound aptamer was determined to be $1.33( \pm 0.36)$, which is $\sim$ half of that for the unbound aptamer. Indeed, target binding can induce a conformational change which presents a fully folded three-dimensional G-quadruplex structure with less flexibility as compared to the free aptamer, thus causing a decrease in the strength of interaction with protamine due to steric hindrance. Although ATP exists mostly as $\mathrm{ATP}^{4-}$ in neutral solution, its electrostatic interaction with protamine is rather weak. Experiments showed that no shifts in the potentiometric response curves were observed for titrations of ATP alone with protamine at concentrations up to $40 \mu \mathrm{M}$. As illustrated in Figure 1C, potentiometric titration with protamine using the traditional protamine-sensitive membrane electrode as an end point detector enables the LFSF aptasensing of ATP down to the submicromolar concentration range.

Selectivity studies show that potential changes are caused exclusively by ATP when compared to other nucleoside triphosphates such as CTP, GTP, and UTP, which suggests the specific recognition of the target analyte by using aptamer as the receptor (Figure S4 in the Supporting Information). The results are consistent with those reported in the literature. ${ }^{31-33}$ It should be noted that the aptamer can be used for monitoring any adenine group containing molecules and therefore cannot differentiate between ATP, ADP, AMP, and adenosine which are different only at the phosphate site (Figure S4 in the Supporting Information). ${ }^{32,38,39}$ Interestingly, another ATP aptamer sequence that can differentiate between ATP and ADP has recently been reported. ${ }^{40}$ With this sequence, a new potentiometric sensor based on the proposed detection strategy can be developed.

Although ATP can be sensitively detected by the potentiometric titration with protamine, the entire titration process might be time-consuming and the electrode response is irreversible (i.e., the electrode is for single use only). Recently, we developed a promising potentiometric biosensing system using pulsed-current-driven reagent delivery for controlledrelease of substrate ions at the sample-membrane interface, which enables rapid, reproducible, and continuous sensing of enzyme activities and screening of their inhibitors and activators. ${ }^{41}$ Herein, we also introduce a unique proteinconditioned polymeric membrane polycation-selective electrode with current-controlled release of protamine from the inner filling solution to the sample solution. The protamine released at the membrane surface can be used as an indicator not only to detect an aptamer but also to report the conformational change of the aptamer upon recognition of a given target, thus offering a novel strategy for developing LFSF potentiometric aptasensors performed in a homogeneous solution (Scheme 1B). The proposed polymeric membrane polycation-selective electrode was constructed as mentioned above. ETH 500 incorporated into the membrane was used to decrease the membrane resistance and thereby the IR voltage drop inside the current-polarized membrane. ${ }^{42}$ Protamine $(0.05$ $\mathrm{mg} / \mathrm{mL}$ ) containing $120 \mathrm{mM} \mathrm{NaCl}$ in $50 \mathrm{mM}$ Tris- $\mathrm{HCl}$ buffer $(\mathrm{pH}$ 7.4) was used as the inner filling and conditioning solutions. It should be noted that conventional polyionsensitive electrodes usually are conditioned with highly discriminated ions and avoid contact with primary ions before measurements to ensure the counterdiffusion of primary ions with discriminated ions. ${ }^{23}$ In contrast, the proposed polycationsensitive electrode is conditioned with the primary ion (i.e., protamine) for generation of ion fluxes of protamine from the inner solution to the sample solution. The strong electrostatic binding interaction between aptamer and protamine facilitates the stripping of protamine out of the membrane surface by the ion-exchange process with discriminated ions from the sample solution.

For precise control of the protamine transportation from the inner solution of the ISE to the sample solution for high reproducibility in potential response, it is essential to apply an external current through the ISE membrane. Higher currents applied can induce larger amounts of protamine released at the sample-membrane interface, which would widen the potential response range but unfortunately decrease the sensitivity for aptamer detection. Therefore, to achieve a lower detection limit and a wider response range, $1 \mu \mathrm{A}$ was chosen as the applied current. Experiments also revealed that the potential response to the aptamer, measured as the difference between the currentdependent potentials for the buffer and sample solutions, could increase with increase in the current pulse duration until $3 \mathrm{~s}$ and 
then remained almost constant. Thus, the optimized current pulse duration was chosen as $3 \mathrm{~s}$.

Under the optimal experimental conditions, the potentiometric response to aptamer is shown in Figure 2. Each pulse of

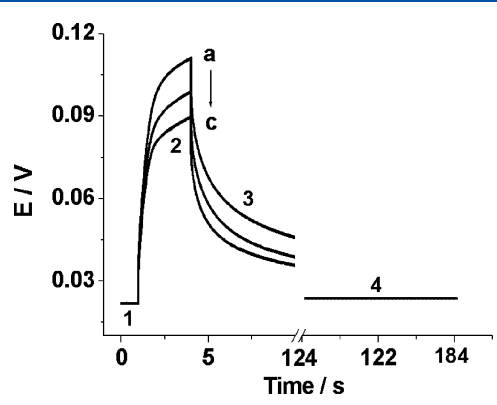

Figure 2. Potentiometric responses of the polycation-sensitive membrane electrode with current-controlled release of protamine in the Tris buffer solutions with 0 (a), 1.67 (b), and $3.33 \mu \mathrm{M}$ aptamer (c). The open-circuit potential of the electrode in Tris buffer was first recorded for $1 \mathrm{~s}$ (pulse 1). An anodic current of $1 \mu \mathrm{A}$ with a duration of $3 \mathrm{~s}$ was then applied across the membrane for controlled-release of protamine (pulse 2), which was followed by a zero current pulse of $120 \mathrm{~s}$ to avoid any undesired IR drop (pulse 3). A controlled voltage at the open-circuit potential of the electrode in the absence of analyte was finally used to refresh the membrane with a recovery time of $60 \mathrm{~s}$ (pulse 4).

the applied current (pulse 2) drives the ion fluxes of protamine through the polymeric membrane and increases the membrane potential. Such current-dependent potential decreases with increasing the concentration of aptamer in the sample solution, which is due to the decrease of the concentration of free protamine released at the sample-membrane interface via the electrostatic binding interaction with aptamer. Notably, the consumption of protamine would facilitate the stripping of protamine out of the membrane surface via the ion-exchange process with sodium ions from the sample solution, which can dramatically decrease the EMF values. Indeed, a similar phenomenon was observed with the conventional polycationsensitive membrane electrode using protamine as the substrate for determination of protease activities. ${ }^{43}$ It should be noted that aptamer can also be detected by using the protamineconditioned polymeric membrane under zero-current conditions. However, it has been found that the spontaneous release of protamine through the ISE membrane would induce a larger potential drift with a longer recovery time ( $\sim 30 \mathrm{~min})$. Since a uniform protamine concentration profile in the membrane phase can be quickly established by applying the external voltage, the membrane of the proposed ISE system is effectively renewed, thus significantly shortening the recovery time (Figure 3).

The potentiometric sensor system using current-driven ion fluxes of protamine was finally examined for aptasensing of ATP. Before potential measurements, ATP was incubated with its aptamer to form the ATP-aptamer complex. As shown in Figure 2, higher concentrations of the aptamer cause larger changes in the current-dependent potentials, but on the other hand would induce lower sensitivity for ATP detection. In this work, $1.67 \mu \mathrm{M}$ of the aptamer was employed to strike a balance between response range and sensitivity.

Figure 4 shows that the presence of the target molecules leads to a binding-inducing conformational change of the aptamer and prevents the aptamer from electrostatically

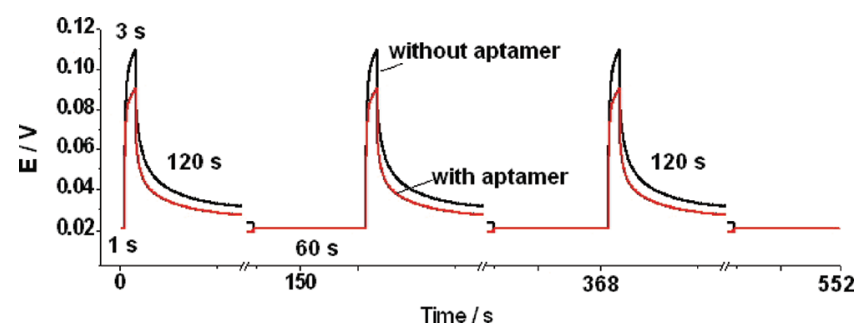

Figure 3. Potential responses of the protamine-conditioned polycation-sensitive membrane electrode to 0.0 (black line) and $3.33 \mu \mathrm{M}$ aptamer (red line) in Tris buffer using the current-driven ion flux strategy.

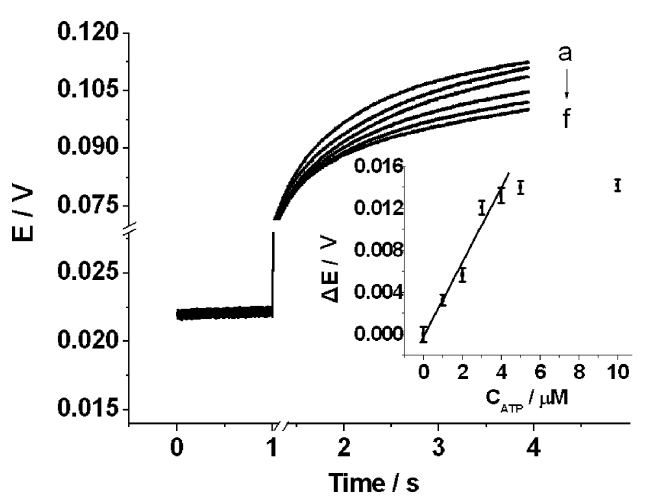

Figure 4. Potentiometric responses of the polycation-sensitive membrane electrode with current-controlled release of protamine to $0.0 \mu \mathrm{M}$ aptamer (a) and $1.67 \mu \mathrm{M}$ aptamer in the presence of $10(\mathrm{~b})$, 3.0 (c), 2.0 (d), 1.0 (e), and $0 \mu \mathrm{M}$ ATP (f) in Tris buffer of $\mathrm{pH}$ 7.4. The inset shows the calibration curve of the membrane electrode for detection of ATP.

interacting with the protamine domain, thus increasing the current-dependent potential. The proposed LFSF aptasensing method using current-driven ion fluxes enables the determination of ATP at concentrations down to $0.31 \mu \mathrm{M}(3 \sigma)$ with a linear concentration range of $0.5-4.0 \mu \mathrm{M}$. In contrast to the conventional ion-selective electrodes which exhibit Nernstian responses, the present polycation-selective electrode shows the sigmoidal relationship between the potential measured and the protamine concentration. ${ }^{44,45}$ At higher concentrations of protamine, the high charge density of protamine causes the potential response function that would be negligibly small. Therefore, the present polycation-sensitive membrane electrode with current-driven release of protamine would be insensitive to free aptamer at lower concentrations. As illustrated in Figure 4, the electrode exhibits nearly saturated potential response at $10 \mu \mathrm{M}$ ATP and further increasing the concentration of ATP could not cause a significant potential change. This may be due to the fact that higher concentrations of ATP consume more free aptamer in the sample solution and the polycation sensor is not sensitive to lower amounts of unbound aptamer. Compared with the response to ATP, negligible changes in the current-dependent potential were observed for its analogue CTP (Figure S5 in the Supporting Information), which is due to the specific recognition of ATP by using its aptamer as discussed above. The detection limit for ATP obtained by the proposed method is comparable to or better than those obtained by other apasensors with fluorescent or amperometric detection. ${ }^{46-48}$ Compare with these systems, the present homogeneous solution-based potentiometirc sensing strategy is much easier to use and needs no labeling, 
separation, and immobilization procedures. In addition, the proposed electrochemical aptasensor can be used in turbid solution.

In order to perform the ATP assay in real samples, the concentration of the cellular ATP was determined by the present membrane electrode with current-driven ion fluxes. As shown in Figure S6 in the Supporting Information, the potential difference between the measurements for the fresh lysate of HeLa cells and the ATP-absent buffer can be clearly observed. The cellular ATP level was calculated as [ATP(cellular) $]=\operatorname{ATP}($ total $) /($ total cell population $\times$ cell volume $)$. By using the cell volume of $2.6 \times 10^{-12} \mathrm{~L},{ }^{49}$ the concentration of the cellular ATP was measured as $0.71 \pm 0.06 \mathrm{mM}$, which is consistent with the ATP concentrations that are typically found in cells $(0.1-3.0 \mathrm{mM}){ }^{50}$ Recently, various fluorescent and electrochemical apatsensors have been developed for cellular ATP assays based on nanoflares, ${ }^{50}$ graphene oxide nanocomplexes, ${ }^{51}$ redox tags, ${ }^{31}$ and copper nanoparticles. ${ }^{52}$ In comparison with these methods, our approach avoids designing complicated aptasensors and allows one to operate in turbid media. It should be noted that the sensitivity of the potentiometric aptasensor can be further improved through appropriate selection of the aptamer with a higher affinity for the target.

\section{CONCLUSIONS}

In conclusion, we have developed a label-free and substrate-free potentiometric platform to signal aptamer/target binding events. In contrast to the conventional approaches of aptasensing, our strategy allows the measurements to be performed in a homogeneous solution, thus effectively eliminating the labeling, separation, and immobilization steps. In addition, the effective potentiometric transduction with polycation-sensitive membrane electrodes suggests that this technique could be operated in turbid media. The proposed potentiometric assay concept can be applied to monitoring other target/aptamer interactions where target binding-induced conformational changes such as the formation of folded structures and the opening of DNA hairpin loops are involved.

\section{ASSOCIATED CONTENT}

\section{S Supporting Information}

Additional information as noted in text. This material is available free of charge via the Internet at http://pubs.acs.org.

\section{AUTHOR INFORMATION}

\section{Corresponding Author}

*Phone: (+86) 535-2109156. Fax: (+86) 535-2109000. E-mail: wqin@yic.ac.cn.

\section{Notes}

The authors declare no competing financial interest.

\section{ACKNOWLEDGMENTS}

This work was financially supported by the National Natural Science Foundation of China (Grant 41176081), the Outstanding Youth Natural Science Foundation of Shandong Province (Grant JQ200814), and the Taishan Scholar Program of Shandong Province (Grant TS20081159).

\section{REFERENCES}

(1) Willner, I.; Zayats, M. Angew. Chem., Int. Ed. 2007, 46, 64086418.
(2) Liu, J. W.; Cao, Z. H.; Lu, Y. Chem. Rev. 2009, 109, 1948-1998.

(3) Mayer, G. Angew. Chem., Int. Ed. 2007, 48, 2672-2689.

(4) Song, S. P.; Wang, L. H.; Fan, C. H.; Zhao, J. L. TrAC, Trends Anal. Chem. 2008, 27, 108-117.

(5) Tang, Z. W.; Mallikaratchy, P.; Yang, R. H.; Kim, Y.; Zhu, Z.; Wang, H.; Tan, W. H. J. Am. Chem. Soc. 2008, 130, 11268-11269.

(6) Mir, M.; Vreeke, M.; Katakis, I. Electrochem. Commun. 2006, 8, $505-511$.

(7) Zhang, Y. L.; Huang, Y.; Jiang, J. H.; Shen, G. L.; Yu, R. Q. J. Am. Chem. Soc. 2007, 129, 15448-15449.

(8) Pavlov, V.; Xiao, Y.; Shlyahovsky, B.; Willner, I. J. Am. Chem. Soc. 2004, 126, 11768-11769.

(9) Xiang, Y.; Xie, M.; Bash, R.; Chen, J. J. L.; Wang, J. Angew. Chem., Int. Ed. 2007, 46, 9054-9056.

(10) So, H. M.; Won, K.; Kim, Y. H.; Kim, B.; Ryu, B. H.; Na, P. S.; Kim, H.; Lee, J. J. Am. Chem. Soc. 2005, 127, 11906-11907.

(11) Li, B. L.; Dong, S. J.; Wang, E. K. Chem. Asian J. 2010, 5, 12621272.

(12) Yeung, M. C. L.; Wong, K. M. C.; Tsang, Y. K. T.; Yam, V. W. W. Analyst 2011, 136, 3268-3273.

(13) Jiang, Y.; Fang, X.; Bai, C. Anal. Chem. 2004, 76, 5230-5235.

(14) Wang, L. H.; Liu, X. F.; Hu, X. F.; Song, S. P.; Fan, C. H. Chem. Commun. 2006, 3780-3782.

(15) Deng, M.; Zhang, D.; Zhou, Y.; Zhou, X. J. Am. Chem. Soc. 2008, 130, 13095-13102.

(16) Du, Y.; Li, B. L.; Wei, H.; Wang, Y. L.; Wang, E. K. Anal. Chem. 2008, 80, 5110-5117.

(17) Numnuam, A.; Chumbimuni-Torres, K. Y.; Xiang, Y.; Bash, R.; Thavarungkul, P.; Kanatharana, P.; Pretsch, E.; Wang, J.; Bakker, E. Anal. Chem. 2008, 80, 707-712.

(18) Zelada-Guill, G. A.; Riu, J.; Dúzgún, A.; Rius, F. X. Angew. Chem., Int. Ed. 2009, 48, 7334-7337.

(19) Ma, S. C.; Yang, V. C.; Meyerhoff, M. E. Anal. Chem. 1992, 64, 694-697.

(20) Ye, Q. S.; Meyerhoff, M. E. Anal. Chem. 2001, 73, 332-336.

(21) Wang, L.; Buchanan, S.; Meyerhoff, M. E. Anal. Chem. 2008, 80, 9845-9847.

(22) Gemene, K. L.; Meyerhoff, M. E. Anal. Chem. 2010, 82, 16121615.

(23) Fu, B.; Bakker, E.; Yun, J. H.; Yang, V. C.; Meyerhoff, M. E. Anal. Chem. 1994, 66, 2250-2259.

(24) Dürűst, N.; Meyerhoff, M. E. J. Electroanal. Chem. 2007, 602, $138-141$.

(25) Esson, J. M.; Meyerhoff, M. E. Electroanalysis 1997, 9, 13251330.

(26) Shvarev, A.; Bakker, E. Anal. Chem. 2005, 77, 5221-5228.

(27) Prieto, M. C.; Maki, A. H.; Balhorn, R. Biochemistry 1997, 36, 11944-11951.

(28) Zhao, Q.; Li, X. F.; Shao, Y. H.; Chris Le, X. Anal. Chem. 2008, $80,7586-7593$.

(29) Cheng, A. K. H.; Ge, B. X.; Yu, H. Z. Anal. Chem. 2007, 79, $5158-5164$.

(30) Xu, Y. D.; Shvarev, A.; Makarychev-Mikhailov, S.; Bakker, E. Anal. Biochem. 2008, 374, 366-370.

(31) Zuo, X. L.; Song, S. P.; Zhang, J.; Pan, D.; Wang, L. H.; Fan, C. H. J. Am. Chem. Soc. 2007, 129, 1042-1043.

(32) Nutiu, R.; Li, Y. F. J. Am. Chem. Soc. 2003, 125, 4771-4778.

(33) Li, N.; Ho, C. M. J. Am. Chem. Soc. 2008, 130, 2380-2381.

(34) Wang, J.; Jiang, Y. X.; Zhou, C. S.; Fang, X. H. Anal. Chem. 2005, 77, 3542-3546.

(35) Ying, Y. L.; Wang, H. Y.; Sutherland, T. C.; Long, Y. T. Small 2011, 7, 87-94.

(36) Shvarev, A.; Bakker, E. J. Am. Chem. Soc. 2003, 125, $11192-$ 11193.

(37) Dai, S.; Meyerhoff, M. E. Electroanalysis 2001, 13, 276-283.

(38) Huizeng, D. E.; Szostak, J. W. Biochemistry 1995, 34, 656-665.

(39) Özalp, V. C. Analyst 2011, 136, 5046-5050.

(40) Sazani, P. L.; Larralde, R.; Szostak, J. W. J. Am. Chem. Soc. 2004, $126,8370-8371$. 
(41) Ding, J. W; Qin, W. J. Am. Chem. Soc. 2009, 131, 14640-14641.

(42) Shvarev, A.; Bakker, E. Anal. Chem. 2003, 75, 4541-4550.

(43) Yun, J. H.; Meyerhoff, M. E. Anal. Biochem. 1995, 224, 212220.

(44) Fordyce, K.; Shvarev, A. Anal. Chem. 2008, 80, 827-833.

(45) Ramamurthy, N.; Baliga, N.; Wahr, J. A.; Schaller, U.; Yang, V. C.; Meyerhoff, M. E. Clin. Chem. 1998, 44, 606-613.

(46) Hu, L. Z.; Bian, Z.; Li, H. J.; Han, S.; Yuan, Y. L.; Xao, G. L.; Xu, G. B. Anal. Chem. 2009, 81, 9807-9811.

(47) Zuo, X. L.; Xiao, Y.; Plaxco, K. W. J. Am. Chem. Soc. 2009, 131, 6944-6945.

(48) Yoshizumi, J.; Kumamoto, S.; Nakamura, M.; Yamana, K. Analyst 2008, 133, 323-325.

(49) Zhao, L.; Kroenke, C. D.; Song, J.; Piwnica-Worms, D.; Ackerman, J. J. H.; Neil, J. J. NMR Biomed. 2008, 21, 159-164.

(50) Zheng, D.; Seferos, D. S.; Giljohann, D. A.; Patel, P. C.; Mirkin, C. A. Nano Lett. 2009, 9, 3258-3261.

(51) Wang, Y.; Li, Z. H.; Hu, D. H.; Lin, C. T.; Li, J. H.; Lin, Y. H. J. Am. Chem. Soc. 2010, 132, 9274-9276.

(52) Zhou, Z. X.; Du, Y.; Dong, S. J. Anal. Chem. 2011, 83, 51225127. 